\title{
Low transmission risk of Pseudomonas aeruginosa in a bronchiectasis clinic based on the knowledge of bacterial population biology
}

\author{
To the Editor:
}

The nosocomial acquisition of opportunistic pathogens, particularly Pseudomonas aeruginosa, has been repeatedly documented in individuals with cystic fibrosis (CF) [1]. Cross-infection is also a concern in bronchiectasis not due to CF [2]. This issue was not addressed by the European Respiratory Society 2017 guidelines for the management of adult bronchiectasis [3] but the European Bronchiectasis Network (EMBARC), the EMBARC Patient Advisory Group and the European Reference Network (ERN-Lung) Bronchiectasis Network released a position statement in the European Respiratory Journal in 2018 [4]. Based on the available evidence, the risk of cross-infection with $P$. aeruginosa was judged to be small.

The position statement explicitly refers to the three most recently published multicentre and single-centre studies, which reported the genotypes of, in total, $704 \mathrm{P}$. aeruginosa isolates collected from 177 patients seen at "non-CF bronchiectasis centres" in the UK [5-7]. Unlike CF, where the Liverpool epidemic strain had spread among the CF community in the UK [8,9], whole-genome sequencing of strains from 16 centres detected no widespread transmissible strain in the UK bronchiectasis community [5]. However, the two single-centre studies identified one duo and one trio among 86 patients who shared strains likely to have been acquired through cross-infection $[6,7]$.

During the last 35 years, we in Hannover (Germany) have set up one of the largest strain collections worldwide of $>5000 P$. aeruginosa isolates, mainly from aquatic habitats in the environment and from human infections, particularly ventilation-associated acute pneumonia and chronic airway infections with COPD or CF [10]. Since our current knowledge of the epidemiology of airway infections with $P$. aeruginosa in non-CF bronchiectasis is based on data gathered in the UK, we reasoned that a further surveillance study in another country backed up by the largest database of transcontinental P. aeruginosa genotypes should provide added value to clarify the issue of cross-infection in bronchiectasis.

From November 2017 to May 2018, all P. aeruginosa isolates that had been retrieved from respiratory secretions of patients attending the local "Bronchiectasis outpatient clinic" were genotyped using a custom-made microarray following the published protocol [11]. This low-resolution microarray represents clone types of the conserved $P$. aeruginosa core genome with 16 informative single-nucleotide polymorphisms and intraclonal variants with 42 marker genes of the variable accessory genome [11].

Of the 143 bronchiectasis patients who were seen during the study period, $P$. aeruginosa was cultured from the respiratory secretions of 49 (34\%) patients. Thus, the prevalence of $P$. aeruginosa-positive patients is significantly higher at our local clinic ( $p=0.006$, Fisher's exact test) than that of $21.7 \%$ calculated from the meta-analysis of 21 observational cohort studies [12]. One patient was co-colonised with two clone types but the other 48 patients were carrying just one clone type in their airways. P. aeruginosa isolates were collected from eight patients on two or more occasions. The microarray always yielded identical genotypes, suggesting that during a short period of 6 months, the turnover of clones is low in bronchiectasis patients.

@ERSpublications

This study suggests that the risk of cross-infection with P. aeruginosa in bronchiectasis is small http:// ow.ly/itUa30nrgET

Cite this article as: Cramer N, Sedlacek L, Tümmler B, et al. Low transmission risk of Pseudomonas aeruginosa in a bronchiectasis clinic based on the knowledge of bacterial population biology. Eur Respir J 2019; 53: 1802191 [https://doi.org/10.1183/13993003.02191-2018]. 
We compared the spectrum of clone types in bronchiectasis patients with those in the global P. aeruginosa populations in the environment, acute pulmonary infection and chronic airway infections in patients with CF (figure 1). $22 \%, 28 \%$ and $24 \%$ of the local bronchiectasis patient population were carrying $P$. aeruginosa clones in their lungs that belong to the 15 most common clones in the environment, acute infection and CF, respectively [10], including the most prevalent clones worldwide, C and PA14 [13]. These proportions of $20-30 \%$ match with results from the adult bronchiectasis service in the north-east of England, which employed the same microarray for strain genotyping [6].

Of the 49 patients, five patient pairs and four patient trios shared the same clone with each other. Thereof, an identical genotype in both core and accessory genome markers was observed in three pairs and two trios, pointing to the possibility that these 12 patients could have acquired their $P$. aeruginosa strains by transmission from shared sources. To clarify this point, we next extracted strain genotypes collected in the CF clinics Munich (Germany) and Hannover from our database. Of 313 isolates collected from $221 \mathrm{CF}$ patients seen at the CF clinic in Munich, 174 strains belonged to clones detected in two patients or more and $77(44 \%)$ strains thereof shared an identical genotype in all markers with at least one other strain. Similarly, of 1112 isolates collected from 134 CF patients from the CF clinic Hannover, the clone type of 191 strains was identified in isolates from two or more patients. 117 (61\%) of the 191 strains shared an identical genotype in all markers of the core and accessory genome with at least one other strain. When we removed the 10 most common $P$. aeruginosa clones that are found worldwide in biotic and abiotic environments [10], 99 strains with shared clone type remained 29 of which having partners with identical genotype. This example demonstrates that knowledge of the population structure of a bacterial species (figure 1) is necessary for the proper interpretation of the abundance and transmission of clones and genotypes.

In the case of the Hannover CF patient population, we had robust epidemiological data to discriminate between transmission and coincidental acquisition of the same genotype. Of the 117 cases of shared $P$. aeruginosa clones, patient-to-patient transmission or nosocomial acquisition from a common source could not be excluded for 36 cases, 17 of which were CF siblings [14]. Of the 19 remaining nosocomial transmissions, 14 cases happened in the 1980s prior to the introduction of patient segregation and hygienic control measures [15] and five cases happened during the last 30 years [16]. These data demonstrate that only a small portion of shared genotypes could be ascribed to nosocomial transmission.

Considering this background, we examined the epidemiology of the cases from the outpatient bronchiectasis clinic with shared genotypes, all of which but one belonged to abundant clones in environment and disease (figure 1) [10]. First of all, all pairs and trios of patients never had an appointment at the same day during the last 4 years. Nine of the 12 patients were already chronically colonised with $P$. aeruginosa at their first visit to the bronchiectasis clinic. Four samples in the study that showed matching $P$. aeruginosa genotypes were collected at this first visit. These data from the patients' records provide strong evidence that none of the 12 patients had acquired $P$. aeruginosa from a source at the bronchiectasis clinic. Most likely, the patients became colonised with $P$. aeruginosa on independent occasions. This interpretation is in line with the conclusion drawn from the genotyping of 2921 isolates

FIGURE 1 Venn diagram of the number of habitat-specific and shared clone types in the Pseudomonas aeruginosa population [10]. Habitats are differentiated by environment (red, 1), acute human infections (green, 2) and chronic airway infections in people with cystic fibrosis (blue, 3) or bronchiectasis (yellow, 4). Numbers in italic indicate the five clone types (hexadecimal code [11] OC2A, EA0A, EC29, F429 and F469) in the local outpatient bronchiectasis population with identical genotypes of core and accessory genome detected by multimarker microarray [11] in two or more patients.

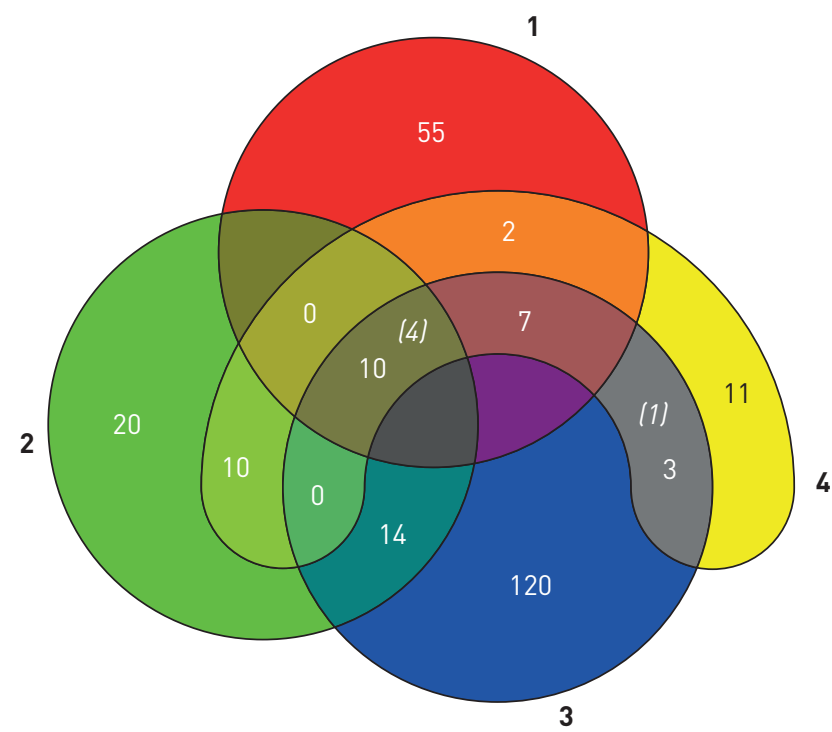


from 1448 independent habitats that the $P$. aeruginosa population is dominated by few epidemic clone types [10]. Nine of the 10 shared genotypes of the bronchiectasis patients belong to the 20 most frequent clones that have an absolute share of $44 \%$ in the population [10].

In summary, shared $P$. aeruginosa genotypes need to be discussed in the context of the bacterial population biology (figure 1) and the patient's records. No evidence for patient-to-patient transmission was identified in this study. Consistent with the position statement [4], our data suggest that the risk of cross-infection with $P$. aeruginosa in bronchiectasis is small.

Nina Cramer ${ }^{1}$, Ludwig Sedlacek ${ }^{2}$, Burkhard Tümmler ${ }^{1,3}$ and Tobias Welte ${ }^{3,4}$

${ }^{1}$ Clinic for Paediatric Pneumology, Allergology and Neonatology, Hannover Medical School, Hannover, Germany.

${ }^{2}$ Institute for Medical Microbiology and Hospital Epidemiology, Hannover Medical School, Hannover, Germany.

${ }^{3}$ Biomedical Research in Endstage and Obstructive Lung Disease (BREATH), German Center for Lung Research, Hannover, Germany. ${ }^{4}$ Dept for Respiratory Medicine, Hannover Medical School, Hannover, Germany.

Correspondence: Burkhard Tümmler, Clinic for Paediatric Pneumology, Allergology and Neonatology, Hannover Medical School, Carl-Neuberg-Str. 1, D-30625 Hannover, Germany. E-mail: tuemmler.burkhard@mh-hannover.de

Received: Nov 162018 | Accepted after revision: Dec 192018

Conflict of interest: N. Cramer has nothing to disclose. L. Sedlacek has nothing to disclose. B. Tümmler reports grants from the Bundesministerium für Bildung und Forschung during the conduct of the study; and personal fees for consultancy and lectures from, and that he has been an investigator in clinical trials for Vertex Pharmaceuticals, and personal fees for lectures from Gilead, outside the submitted work. T. Welte reports grants from the Bundesministerium für Bildung und Forschung during the conduct of the study; and personal fees from AstraZeneca and Basilea, Infectofarm, Boehringer, Berlin Chemie, Mundipharma and Insmed, and grants and personal fees from Bayer, Grifols, Novartis and Pfizer, outside the submitted work.

Support statement: This study was supported by Bundesministerium für Bildung und Forschung (grant 82DZL002A1). Funding information for this article has been deposited with the Crossref Funder Registry.

\section{References}

1 Parkins MD, Somayaji R, Waters VJ. Epidemiology, biology, and impact of clonal Pseudomonas aeruginosa infections in cystic fibrosis. Clin Microbiol Rev 2018; 31: e00019-18.

2 Mitchelmore P, Wilson C, Hettle D. Risk of bacterial transmission in bronchiectasis outpatient clinics. Curr Pulmonol Rep 2018; 7: 72-78.

3 Polverino E, Goeminne PC, McDonnell MJ, et al. European Respiratory Society guidelines for the management of adult bronchiectasis. Eur Respir J 2017; 50: 1700629.

4 Chalmers JD, Ringshausen FC, Harris B, et al. Cross-infection risk in patients with bronchiectasis: a position statement from the European Bronchiectasis Network (EMBARC), EMBARC/ELF patient advisory group and European Reference Network (ERN-Lung) Bronchiectasis Network. Eur Respir J 2018; 51: 1701937.

5 Hilliam Y, Moore MP, Lamont IL, et al. Pseudomonas aeruginosa adaptation and diversification in the non-cystic fibrosis bronchiectasis lung. Eur Respir J 2017; 49: 1602108.

6 De Soyza A, Perry A, Hall AJ, et al. Molecular epidemiological analysis suggests cross-infection with Pseudomonas aeruginosa is rare in non-cystic fibrosis bronchiectasis. Eur Respir J 2014; 43: 900-903.

7 Mitchelmore PJ, Randall J, Bull MJ, et al. Molecular epidemiology of Pseudomonas aeruginosa in an unsegregated bronchiectasis cohort sharing hospital facilities with a cystic fibrosis cohort. Thorax 2018; 73: 677-679.

8 Fothergill JL, Walshaw MJ, Winstanley C. Transmissible strains of Pseudomonas aeruginosa in cystic fibrosis lung infections. Eur Respir J 2012; 40: 227-238.

9 Williams D, Fothergill JL, Evans B, et al. Transmission and lineage displacement drive rapid population genomic flux in cystic fibrosis airway infections of a Pseudomonas aeruginosa epidemic strain. Microb Genom 2018; 4: https://doi.org/10.1099/mgen.0.000167.

10 Wiehlmann L, Cramer N, Tümmler B. Habitat-associated skew of clone abundance in the Pseudomonas aeruginosa population. Environ Microbiol Rep 2015; 7: 955-960.

11 Wiehlmann L, Wagner G, Cramer N, et al. Population structure of Pseudomonas aeruginosa. Proc Natl Acad Sci USA 2007; 104: 8101-8106.

12 Finch S, McDonnell MJ, Abo-Leyah $\mathrm{H}$, et al. A comprehensive analysis of the impact of Pseudomonas aeruginosa colonization on prognosis in adult bronchiectasis. Ann Am Thorac Soc 2015; 12: 1602-1611.

13 Fischer S, Klockgether J, Morán Losada P, et al. Intraclonal genome diversity of the major Pseudomonas aeruginosa clones C and PA14. Environ Microbiol Rep 2016; 8: 227-234.

14 Grothues D, Koopmann U, von der Hardt H, et al. Genome fingerprinting of Pseudomonas aeruginosa indicates colonization of cystic fibrosis siblings with closely related strains. J Clin Microbiol 1988; 26: 1973-1977.

15 Tümmler B, Koopmann U, Grothues D, et al. Nosocomial acquisition of Pseudomonas aeruginosa by cystic fibrosis patients. J Clin Microbiol 1991; 29: 1265-1267.

16 Wiehlmann L, Cramer N, Ulrich J, et al. Effective prevention of Pseudomonas aeruginosa cross-infection at a cystic fibrosis centre - results of a 10-year prospective study. Int J Med Microbiol 2012; 302: 69-77. 